\title{
Quantitative Attenuation Correction for PET/CT Using Iterative Reconstruction of Low-Dose Dual-Energy CT
}

\author{
Paul E. Kinahan, Senior Member, IEEE, Jeffrey A. Fessler, Senior Member, IEEE, \\ Adam M. Alessio, Member, IEEE, and Thomas K. Lewellen, Senior Member, IEEE \\ University of Washington Medical Center, Seattle, WA
}

\begin{abstract}
We present the results of using iterative reconstruction of dual-energy CT (DECT) to perform accurate CT-based attenuation correction (CTAC) for PET emission images. Current methods, such as bilinear scaling, introduce quantitative errors in the PET emission image for bone, metallic implants, and contrast agents. DECT has had limited use in the past for quantitative CT imaging due to increased patient dose and high noise levels in the decoupled CT basis-material images. Reconstruction methods that model the acquisition physics impose a significant computational burden due to the large image matrix size (typically 512 x 512). For CTAC, however, three factors make DECT feasible: (1) a smaller matrix is needed for the transmission image, which reduces the noise per pixel, (2) a smaller matrix significantly accelerates an iterative CT reconstruction algorithm, (3) the monoenergetic transmission image at $511 \mathrm{keV}$ is the sum of the two decoupled basis-material images. Initial results using a $128 \times 128$ matrix size for a test object comprised of air, soft tissue, dense bone, and a mixture of tissue and bone demonstrate a significant reduction of bias using DECT (from $20 \%$ to $\sim 0 \%$ for the tissue/bone mixture). FBP reconstructed images, however, have significant noise. Noise levels are reduced from $\sim 8 \%$ to $\sim 3 \%$ by the use of PWLS reconstruction.
\end{abstract}

\section{INTRODUCTION}

$\mathrm{D}^{\mathrm{ET} / \mathrm{CT} \text { scanners are used to provide accurately aligned }}$ functional and anatomical information [1]. A secondary synergy of PET/CT scanners is to use the CT image for attenuation correction of the PET emission data [2]. CT-based attenuation correction (CTAC) offers several benefits over conventional PET transmission scan attenuation correction. CTAC provides low-noise attenuation correction factors without the need for lengthy transmission scans, significantly reducing the overall scan time. Moreover, CT scans contain

P.E. Kinahan, A.M. Alessio and T.K. Lewellen are with the Department of Radiology, University of Washington, Seattle, WA 98195 USA (telephone: 206-598-4521 email: kinahan@u.washington.edu)

J. A. Fessler is with the Electrical Engineering and Computer Science Department, University of Michigan, Ann Arbor, MI 48109-2122 USA (email: fessler@umich.edu)

This work supported by NIH grants CA74135 and CA42593

no bias from the emission contamination of post-injection imaging.

CT attenuation correction factors are susceptible to bias due to the need to transform the CT image to attenuation correction factors at $511 \mathrm{keV}$. CTAC biases are probably not significant for detection or staging purposes [3], but are problematic with prosthetics [4] or where quantitative accuracy is paramount. Figure 1 summarizes the benefits and challenges of CTAC.

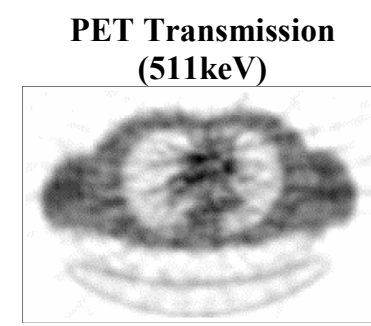

high noise

$\sim 12-45$ min scan time no bias

lowest contrast post injection contamination

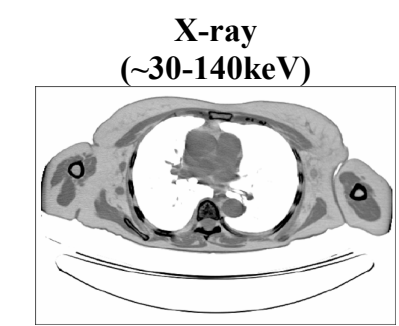

no noise $\sim 20$ sec scan time potential for significant bias high contrast no post injection contamination

Fig 1. Summarizes the pros and cons of common methods for attenuation correction.

\section{CT-BAsed AtTENUATION CORRECTION}

Three simple solutions can be employed for transforming attenuation levels from CT energies to PET energy. Segmentation methods can be used to separate the CT image into regions corresponding to different tissue types, which then are replaced with appropriate attenuation coefficients at 511 $\mathrm{keV}$. This method has a potential source of error due to misclassification, especially with low-dose CTAC, and in areas with low density gradients although this approach may be helpful for focal contrast accumulation $[5,6]$. Second, linear scaling of the entire CT image with the ratio of attenuation coefficients of water (representing soft tissues) at the photon 
energies of CT and PET offers a simple solution for the transformation. For bone, however, linear scaling is a poor approximation, since photoelectric absorption dominates Compton scatter at the lower range of CT energies [7]. Finally, the most common method for transforming to PET energies is Bilinear/Hybrid Scaling. In these methods, different scaling factors (for water and air, and for water and bone respectively) are used to calculate the attenuation values for CT numbers $\mathrm{H}$ for which $-1000<\mathrm{H}<0$, and for $\mathrm{H}>0$. The bilinear approach was proposed for PET [8] and [9] and a variation of bilinear method, hybrid scaling, was proposed in [2]. These methods make approximations that include treating the CT energy spectrum as a single 'effective' energy and ignoring the atomic number of high- $Z$ materials such as contrast agents [10].

Both the bilinear scaling method and the hybrid methods have been shown to give reasonable results for low-Z biological materials in practice. However, for high- $Z$ materials such as contrast agent there is a possibility for significant bias. Figure 2 presents reconstructions of a cylindrical phantom containing water and three small cylinders of air, a solution of iodine-based contrast agent, and a solution of $\mathrm{CaCl} 2$ (a bone equivalent mixture). Figure $2 \mathrm{C}$ shows the same phantom $\mathrm{PET}$ transmission scan revealing the correct attenuation values for $511 \mathrm{keV}$ photons. Figure $2 \mathrm{D}$ is the difference image of the bilinear scaled image and the PET transmission image highlighting the significant errors in the contrast cylinder.

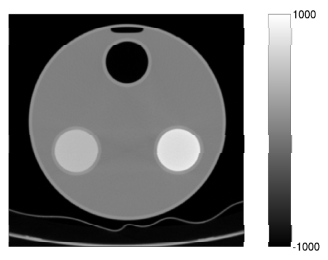

A. Original $140 \mathrm{keV}$ CT Image

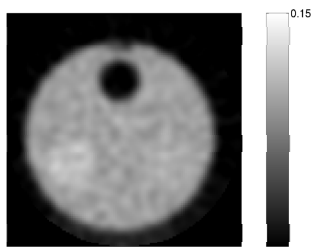

C. PET Tx Image

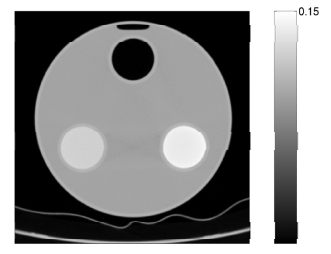

B. Downsampled and bilinear scaled image

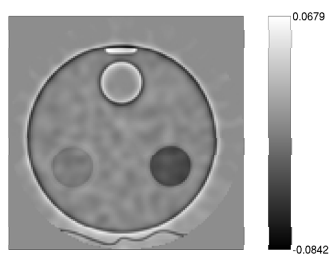

D. Difference image of B and C Fig 2. Highlights the potential bias due to bilinear scaling of CT image. Figure (A) shows original CT image of water phantom with air, contrast, and bone cylinders starting at top and proceeding clockwise. Images (B) and (C) show linear attenuation coefficient values at $511 \mathrm{keV}$ from bilinear scaling of CT image and from PET Tx scan. Difference image in (D) shows most error in cylinder containing contrast agent.

Biases in the attenuation correction factors lead to errors in the attenuation corrected PET images. These errors are difficult to predict and lead to images with inaccurate quantitation and boundary artifacts. Figure 3 presents a simulated example highlighting the propagation of errors in attenuation values into errors in PET images. Figure 4 plots the error in the attenuation corrected PET standard uptake values (SUV) for different levels of errors in the CT images. As the error in the ACF's increase, the error in the PET image increases and regions with higher uptake values lead to greater errors in the AC PET image.
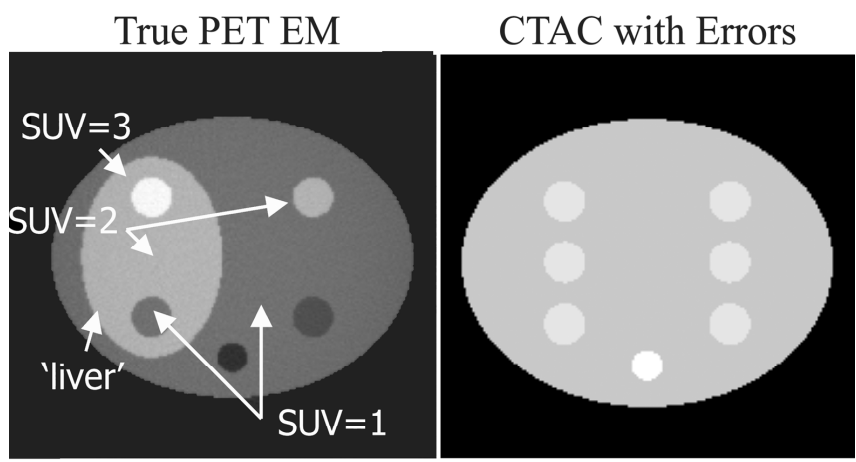

PET EM using CTAC Difference in PET images
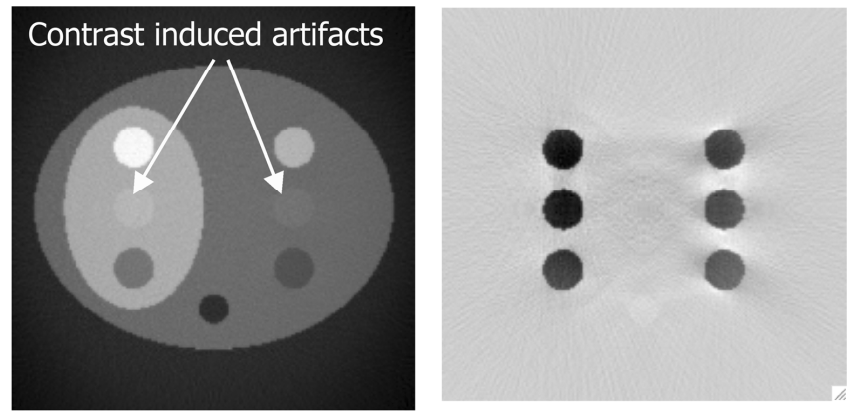

Fig 3. Simulation study showing propagation of errors from biased attenuation correction factors (CTAC with errors) into the attenuation corrected PET image (PET EM using CTAC). Difference image shows that both quantitative errors can occur along with unpredictable artifacts.

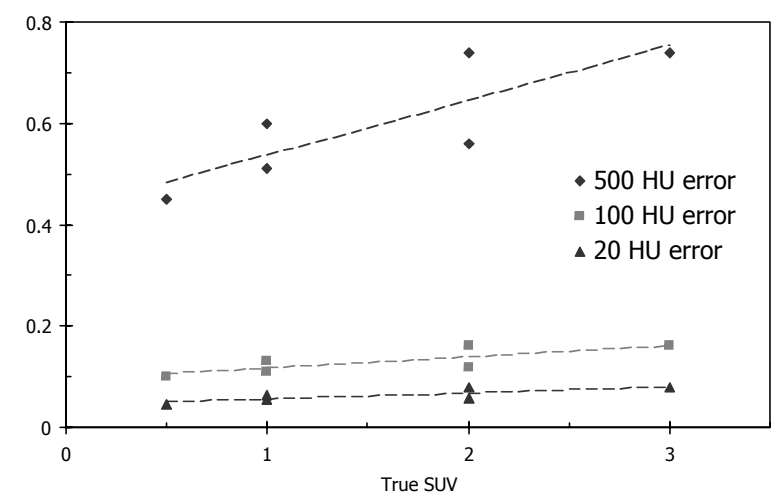

Fig 4. Results of simulation study showing propagation of CT error of different Hounsfield units (HU) into the error in the attenuation corrected PET standard uptake values (SUV). 


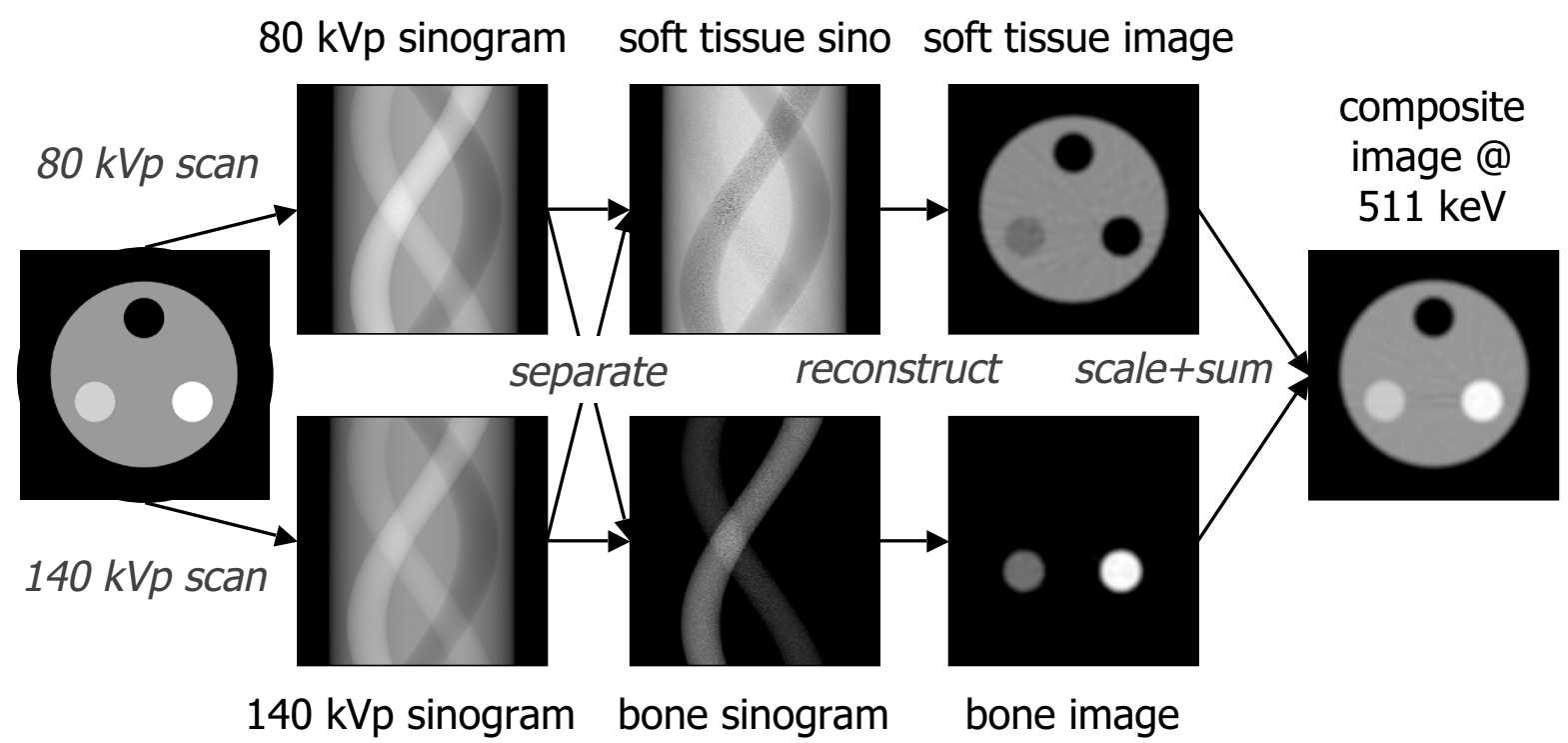

Fig 5. Schematic of the steps in dual energy CT-based, attenuation correction. The object is initially scanned at two energies. Sinograms are then decomposed into basis sinograms of soft tissue and bone. The soft tissue and bone images are reconstructed (with FBP in this case) from these separate sinograms. Finally, the separate images are scaled to $511 \mathrm{keV}$ and combined into composite image for attenuation correction of the PET emission data.

\section{DuAl ENERGy CT-BASEd ATtENUATION CORRECTION}

We propose using dual energy CT (DECT) [11] to remove the bias from the CTAC image. This approach was proposed with SPECT [12] and PET [13]. DECT is problematic because of the significant noise amplification and the additional patient radiation dose required to perform second scan and to reduce noise. Figure 5 summarizes the steps involved in dual energy CT-Based attenuation correction. Our goal is to use dual energy CT for PET attenuation correction with a statisticallyprincipled reconstruction method to reduce noise and with a smaller grid size to make iterative reconstruction feasible. Iterative $\mathrm{CT}$ reconstruction has received considerable interest, but no clinical application due to the computation time for large (e.g 512 $2^{2}$ image sizes. For dual energy CTAC, high resolution $\mathrm{CT}$, and consequently large image sizes, are not required considering the PET images are at a much lower resolution. As a result, we can reduce the computational burden of iterative reconstruction to a clincally feasible level.

\section{A. Dual Energy CT Attenuation Correction with PWLS}

Sukovic and Clinthorne proposed a PWLS algorithm for use with DECT data [14]. Our method is an extension using a polychromatic X-ray spectra for the decomposition of the dual energy sinograms into soft tissue and bone sinograms. Figure 6 presents the two energy spectra used in the simulations.

Starting with decomposed sinograms, $\hat{\mathbf{s}}=\left[\hat{s}_{S}, \hat{s}_{B}\right]$, for soft tissue and bone basis materials, the PWLS similarity measure is

$$
\Psi(\mathbf{x})=\sum_{i=1}^{N_{d}} \frac{1}{2}\left(\hat{\mathbf{s}}_{i}-\mathbf{s}_{i}(\mathbf{x})\right)^{\prime} \mathbf{W}_{i}\left(\hat{\mathbf{s}}_{i}-\mathbf{s}_{i}(\mathbf{x})\right)+\beta R(\mathbf{x})
$$

where $i$ is one of $N_{d}$ detector bins in $\hat{s}_{S} . \quad \mathbf{s}_{i}(\mathbf{x})$ is the forward projection of images $\mathbf{x}$ such that $\mathbf{s}_{i}(\mathbf{x}) \triangleq\left[s_{S i}\left(x_{S}\right), s_{B i}\left(x_{B}\right)\right]$ where

$$
s_{S i}\left(x_{S}\right)=\sum_{j=1}^{N_{p}} a_{i j} x_{S j} .
$$

The contribution of pixel $j$ to sinogram bin $i$ is $a_{i j}$ found from

$$
a_{i j}=\int_{\mathrm{L}_{i}} b_{j}(x) d l
$$

the line integral of spatial basis function $b_{j}(x)$.

The weighting matrix, $\mathbf{W}_{i}$ is a $2 \times 2$ matrix. In this paper, we choose to let $\mathbf{W}_{i}$ be diagonal with

$$
\mathbf{W}_{i}=\left(\begin{array}{cc}
\frac{1}{\sigma_{S i}^{2}} & 0 \\
0 & \frac{1}{\sigma_{B i}^{2}}
\end{array}\right)
$$

where $\sigma_{i s}^{2}$ is an estimate of the variance of sinogram element $\hat{s}_{S i}$. The off-diagonal terms can be used to include the wellknown covariance between the basis sinograms. The penalty term $R(\mathbf{x})$ is regulated with strength, $\beta$. The images $\mathbf{x}=\left[x_{S}, x_{B}\right]$ that minimize (1) provide the PWLS estimate of the coefficients we desire [15].

The desired attenuation coefficients at any energy are

$$
\mu(x, E)=\sum_{l=1}^{2} \sum_{j=1}^{N_{p}} \beta_{l}(E) b_{j}(x) x_{l j}
$$


where $\beta_{l}(E)$ is the mass attenuation coefficient for material $l$ where $l=[1,2]$.

\section{APPLICATION}

Figure 7 presents a comparison of the reconstruction of soft tissue and bone images with FBP and PWLS. Data from this simulated chest phantom was generated with the $80 \mathrm{kVp}$ and $140 \mathrm{kVp}$ polychromatic spectra in figure 6 . The PWLS method results in less error and noise in the images.

We also simulated a NEMA-94 $20 \mathrm{~cm}$ test cylinder with three $5 \mathrm{~cm}$ inserts containing air, 50-50 soft tissue + bone and $100 \%$ bone. To evaluate the proposed DE polychromatic PWLS at varying image and data sizes, we generated 20 independent noise realizations of dual energy data sets from this phantom. These data sets were reconstructed to images with matching spatial resolution, as desired for determining PET attenuation correction factors. Table 1 summarizes the results providing the time per iteration, RMS error, and average bias. It should be stressed that smaller image sizes still lead to accurate reconstructions with comparable errors and bias as the high resolution reconstructions. Figure 8 plots the reconstruction time of PWLS and FBP vs. the data and image size reduction factor. Even though these times are based on non-optimized Matlab code, the plot highlights that iterative reconstruction of smaller image sizes can be accomplished in a reasonable amount of time.
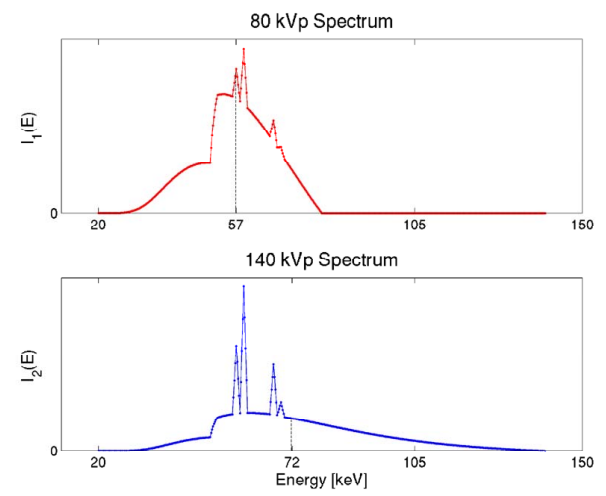

Fig 6. Simulated energy spectra used to decompose dual energy data into soft tissue and bone components.

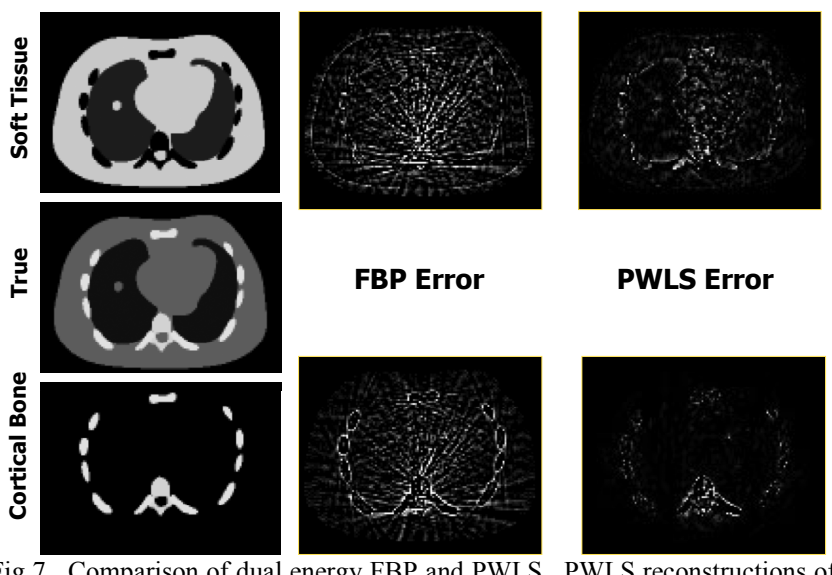

Fig 7. Comparison of dual energy FBP and PWLS. PWLS reconstructions of soft tissue and bone components have less error and noise than FBP reconstructions.

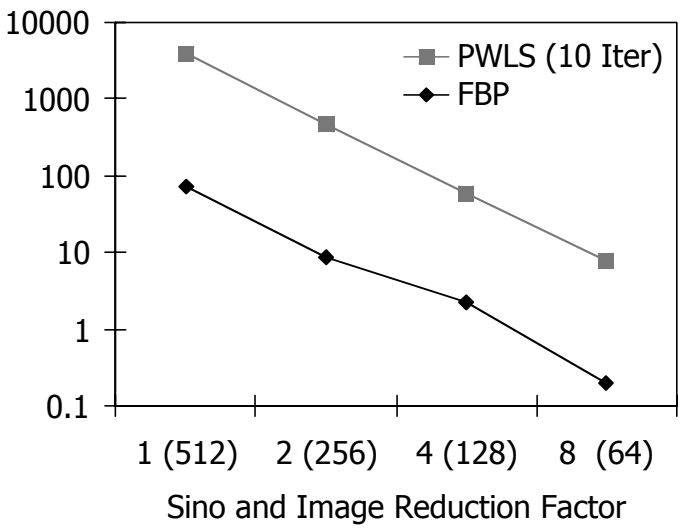

Fig 8. Time comparison of FBP and PWLS as the image and data size is reduced by factors 1 through 8 . Shows that PWLS reconstruction of small images can be accomplished in feasible times.

TABLE 1. COMPARISON OF DE-PWLS RECONSTRUCTION OF DIFFERENT IMAGE/DATA SIZES.

\begin{tabular}{|c|c|c|c|c|}
\hline $\begin{array}{c}\text { Image } \\
\text { Size }\end{array}$ & $\begin{array}{c}\text { Sinogram } \\
\text { Size }\end{array}$ & $\begin{array}{l}\text { Time per it } \\
\text { relative to } \\
512 \times 512 \\
\text { FBP }\end{array}$ & $\begin{array}{l}\text { Normalized } \\
\text { RMS Error }\end{array}$ & $\begin{array}{c}\text { Average } \\
\text { absolute } \\
\text { bias }\end{array}$ \\
\hline $512 \times 512$ & $560 \times 640$ & 5.3 & $20.3 \pm 0.1 \%$ & $11.1 \pm 0.2 \%$ \\
\hline $128 \times 128$ & $140 \times 160$ & 0.1 & $13.7 \pm .42 \%$ & $13.9 \pm 0.6 \%$ \\
\hline
\end{tabular}

\section{CONCLUSION}

Currently, CT-based attenuation correction has the potential for significant bias in the attenuation correction factors. We present results showing the propagation of these errors into attenuation corrected PET images. Dual energy CT-based attenuation correction offers the potential of reducing bias in the CTAC image and also bias in the final PET emission image. Dual energy CT results in increased noise in 
reconstructions from the basis material decomposition process and from the potential use of low-dose CT. Noise from dual energy CT imaging can be reduced through the use of 1) a statistically principled reconstruction method, 2) a coarse reconstruction grid, and 3) only the summed component image at $511 \mathrm{keV}$.

We presented a successful polychromatic, DE-PWLS iterative reconstruction method. We showed that the reduction of the CTAC sinograms and image matrix sizes to $128 \times 128$ or $64 \times 64$ leads to iterative reconstructions of dual energy CT component images in a clinically feasible amount of time.

\section{REFERENCES}

[1] T. Beyer, D. Townsend, T. Brun, P. Kinahan, M. Charron, R. Roddy, J. Jerin, J. Young, L. Byars, and R. Nutt, "A combined PET/CT scanner for clinical oncology," J Nucl Med, vol. 41, pp. 1369-1379, 2000.

[2] P. E. Kinahan, D. W. Townsend, T. Beyer, and D. Sashin, "Attenuation correction for a combined 3D PET/CT scanner," Med Phys, vol. 25, pp. 2046-53, 1998.

[3] E. Dizendorf, T. F. Hany, A. Buck, G. K. von Schulthess, and C. Burger, "Cause and Magnitude of the Error Induced by Oral CT Contrast Agent in CT-Based Attenuation Correction of PET Emission Studies," J Nucl Med, vol. 44, pp. 732-738, 2003.

[4] G. W. Goerres, C. Burger, E. Kamel, B. Seifert, A. H. Kaim, A. Buck, T. C. Buehler, and G. K. von Schulthess, "Respiration-induced Attenuation Artifact atPET/CT: Technical Considerations," Radiology, vol. 226, pp. 906-910, 2003.

[5] J. Carney, T. Beyer, D. Brasse, J. T. Yap, and D. W. Townsend, "CTbased attenuation correction for PET/CT scanners in the presence of contrast agent," presented at Nuclear Science Symposium Conference Record, 2002 IEEE, 2002.

[6] S. A. Nehmeh, Y. E. Erdi, H. Kalaigian, K. S. Kolbert, T. Pan, H. Yeung, O. Squire, A. Sinha, S. M. Larson, and J. L. Humm, "Correction for Oral Contrast Artifacts in CT Attenuation-Corrected PET Images Obtained by CombinedPET/CT," J Nucl Med, vol. 44, pp. 1940-1944, 2003.

[7] K. J. LaCroix, B. M. W. Tsui, B. H. Hasegawa, and J. K. Brown, "Investigation of the use of X-ray CT images for attenuation compensation in SPECT," IEEE Trans Nuclear Science, vol. 41, pp. 2793-2799, 1994.

[8] C. Burger, G. Goerres, S. Schoenes, A. Buck, A. H. Lonn, and G. K. Von Schulthess, "PET attenuation coefficients from CT images: experimental evaluation of the transformation of CT into PET $511-\mathrm{keV}$ attenuation coefficients," Eur J Nucl Med Mol Imaging, vol. 29, pp. 922-7, 2002.

[9] C. Bai, L. Shao, A. J. Da Silva, and Z. Zhao, "A generalized model for the conversion from CT numbers to linear attenuation coefficients," IEEE Trans Nuclear Science, vol. 50, pp. 1510-1515, 2003.

[10] P. E. Kinahan, B. H. Hasegawa, and T. Beyer, "X-ray-based attenuation correction for positron emission tomography/computed tomography scanners," Semin Nucl Med, vol. 33, pp. 166-179, 2003.

[11] R. E. Alvarez and A. Macovski, "Energy-selective reconstructions in Xray computerized tomography," Phys Med Biol, vol. 21, pp. 733-44, 1976.

[12] K. L. Goh, S. C. Liew, and B. H. Hasegawa, "Correction of energydependent systematic errors in dual-energy x-ray CT using a basis material coefficients transformation method," IEEE Transactions on Nuclear Science, vol. 44, pp. 2419-2424, 1997.

[13] M. J. Guy, I. A. Castellano-Smith, M. A. Flower, G. D. Flux, R. J. Ott, and D. Visvikis, "DETECT-dual energy transmission estimation CT-for improved attenuation correction in SPECT and PET," IEEE Trans Nuclear Science, vol. 45, pp. 1261-1267, 1998.

[14] P. Sukovic and N. H. Clinthorne, "Penalized weighted least-squares image reconstruction for dual energy X-ray transmission tomography," Medical Imaging, IEEE Transactions on, vol. 19, pp. 1075-1081, 2000.

[15] J. A. Fessler, "Penalized Weighted Least-Squares Image Reconstruction for Positron Emission Tomography," IEEE Trans Med Imaging, vol. 13, pp. 290-299, 1994. 